RESEARCH REPORT

\title{
Sense of coherence as a moderator of the effects of stressful life events on health
}

\author{
Chris G Richardson, Pamela A Ratner
}

J Epidemiol Community Health 2005;59:979-984. doi: 10.1136/jech.2005.036756

See end of article for authors' affiliations .....................

Correspondence to: Dr C G Richardson, School of Nursing, 302-6190 Agronomy Road, University of British Columbia, Vancouver, BC, Canada V6T 1Z3; richardson_cg@yahoo. com

Accepted for publication 15 July 2005
Study objective: To test the hypothesis that Antonovsky's concept of sense of coherence (SOC) moderates (that is, buffers) the health impacts of stressful life events in a population based sample.

Design: Multiple linear and Poisson regression analyses of longitudinal data from a national survey of population health were used to examine the relations among SOC, the experience of recent stressful life events (for example, family breakdown, financial crisis, physical abuse), and two outcomes, self reported health status (SRH) and self reported number of physician visits during the previous year.

Setting: General population of Canada.

Participants: Nationally representative sample of household residents aged 30 years of age or greater surveyed in 1998 and $2000(n=6505)$.

Main results: After controlling for age, sex, and previous health status, a significant moderating effect $(t=2.24, p=0.025)$ in the expected direction was found on respondents' SRH. The mean difference in SRH between those who did and did not experience a recent stressful life event was 0.24 (95\% Cl: $0.16,0.32$ ) in people with a below average SOC and $0.04(95 \% \mathrm{Cl}:-0.04,0.11)$ in people with a higher than average SOC score. The postulated moderating effect of SOC was not significant $(t=1.1, p>0.05)$ in predicting the number of visits to a physician.

Conclusions: SOC seems to buffer the impact of recent stressful life events on SRH.
A substantial amount of research has been devoted to the study of psychosocial characteristics and their relations with stress and health. Although early studies focused on the effects of differential exposures to stress, researchers soon realised that substantial gains in understanding the relation could be obtained by taking into account systematic differences in vulnerability to the impact of stress. ${ }^{1}$ Prominent among the protective theories, Antonovsky's concept of sense of coherence (SOC) emerged as part of a theory of salutogenesis that attempts to explain and predict movement toward the healthy end of the health ease/dis-ease continuum. ${ }^{23}$ According to Antonovsky, stressors in people's lives are endemic, ubiquitous, and open ended (positive or negative).$^{4}$ Within such a paradigm, the focus of investigation shifts from the investigation of particular stressors (for example, work stressors) to the study of more general salutary factors (capabilities) that enable people to adapt to the endless and diverse array of challenges encountered.

Broader than a specific coping mechanism, Antonovsky theorised that SOC represents a higher level distillation of the common components underlying particular coping or resistance resources (for example, social support, education, and financial resources).$^{3-5}$ It is:

A global orientation that expresses the extent to which one has a pervasive, enduring though dynamic feeling of confidence that (1) the stimuli, deriving from one's internal and external environments in the course of living are structured, predictable and explicable; (2) the resources are available to one to meet the demands posed by these stimuli; and (3) these demands are challenges, worthy of investment and engagement. $^{3}$

These three components were labelled: comprehensibility (the extent of the belief that the problem faced by the person is clear), manageability ( the extent of the belief that not only does one understand the problem, but that the necessary resources to successfully cope with the problem are available), and meaningfulness (the extent of the belief that coping "makes sense" emotionally, that one wishes to cope). Although these components were identified as distinct, albeit related components, SOC represents a single, higher order generalised orientation.

A person's SOC is said to develop through childhood into the latter stages of adolescence. During this time, people are repeatedly exposed to tension states requiring that they actively respond to stressors by mobilising appropriate resources. ${ }^{3}$ Antonovsky speculated that a person's SOC increases the degree to which tension states are perceived as comprehensible, manageable, and meaningful thereby reducing the potential for psychological distress. ${ }^{3}$ Over time, the repeated and healthful management of tension events reinforces the person's SOC and increases the coping resources available in the future. Antonovsky further theorised that by about the end of their third decade, people will have been exposed to a sufficiently long and consistent pattern of experiences that their SOC becomes a stable dispositional orientation. ${ }^{3}$

The relations predicted by the theory of salutogenesis have been examined in several populations. In an investigation of health protective factors in older adults, Lutgendorf et al found that SOC moderated the relation between being relocated to independent congregate living facilities (yes or no) and immune functioning. ${ }^{6}$ In a cross sectional study of the relation between the psychosocial work environment and stress symptoms, Albertsen et al reported significant moderating by SOC. ${ }^{7}$ However, the effect was significant in only 5 of the 25 environmental factor-stress symptom relations investigated. Feldt reported that employees with a strong SOC

Abbreviations: SOC, sense of coherence; $\mathrm{SRH}$, self reported health; RLE, recent stressful life event; VIF, variance inflation factor 
were better protected from the adverse psychological effects of certain work conditions than employees with a weak SOC. ${ }^{8}$

Because a person's SOC putatively buffers the impact of stressful life events (that is, it facilitates the selection of appropriate and efficacious coping resources and behaviour in addition to increasing the extent to which tension states are perceived as comprehensible, manageable, and meaningful), ${ }^{3}$ the following hypothesised effect of SOC was tested: SOC moderates the impact of recent stressful life events on self reported health (SRH) status and number of self reported visits to a medical doctor.

\section{METHODS \\ Data}

Since 1994, Statistics Canada has conducted the biennial, longitudinal national population health survey (NPHS) to assess the level, distribution, and trend of the health status of the Canadian population, and to provide data that reflect the dynamic processes of health and illness. ${ }^{9}$ The sampling design for the NPHS is that of multistage stratified cluster design. The survey includes household residents in all provinces of Canada with the exception of populations on Indian Reserves and Crown Lands, residents of healthcare institutions, members of the Canadian Armed Forces, and those in some remote areas in Ontario and Quebec. ${ }^{10}$ Sample weights reflecting the probability of a person being selected for inclusion in the initial wave of the survey are calculated.

The longitudinal data analysed in this study were collected in 1998 and 2000. The response rate for the initial 1994 survey was $83.6 \%$ and the 2000 follow up response rate was $84.8 \%$. The sample of 6517 cases examined in this study was formed by selecting respondents aged 30 years of age and greater, in 1994, with no missing data on the relevant variables. Of these cases, 12 were deleted because their reported number of medical visits in the previous year exceeded 100 visits.

\section{Instruments}

\section{Sense of coherence scale}

The 13 item SOC scale was included in the NPHS. ${ }^{5}$ Responses to each question were scored on a Likert type scale ranging from 1 to 7 with higher scores showing a stronger SOC. ${ }^{11}$ Cronbach's $\alpha$ for the 13 item SOC Scale have ranged from 0.74 to $0.91 . .^{5}$

\section{Adjusted recent life events index}

Included in the 2000 NPHS survey were questions about the number of negative events the respondent or someone close to the respondent experienced in the past 12 months. The adjusted recent life events index included: (a) physical abuse, (b) unwanted pregnancy, (c) abortion or miscarriage, (d) major financial crisis, (e) serious problems in school, (f) change of job for a worse one, (g) demotion or cut in pay, and (h) going on welfare. For partnered persons (married or living common law), the index included a question (i) about increased arguments with the respondent's partner. For persons who had children, the index included $(\mathrm{j})$ a question about children moving back home. For each positive response, respondents were awarded one point with the total number of positive responses varying from 8 to 10 depending on social role (that is, whether they were partnered or had children). ${ }^{11}$ To create the adjusted recent life events index, the responses were summed and adjusted as if the 10 questions were relevant to all the respondents (possible scores ranged 0 to 10). A higher score shows greater exposure to stressful life events in the previous 12 months.

\section{Visits to a medical doctor}

The number of visits to a medical doctor in the past 12 months was assessed by asking, "In the past 12 months, how many times have you seen or talked on the telephone about your physical, emotional or mental health with a family doctor, paediatrician or general practitioner?"11 The respondent was prompted to consider visits with "any other medical doctor (such as a surgeon, allergist, orthopaedist, gynaecologist, or psychiatrist)."

\section{Self reported health status}

SRH status was assessed by asking respondents to rate their health as: (1) poor, (2) fair, (3) good, (4) very good, or (5) excellent. Research has shown that the item correlates strongly with other direct and indirect measures of health including the sickness impact profile and subscales of the short form 36 health survey. ${ }^{12}{ }^{13}$ Research also has shown that the SRH item is an efficient and valid means of assessing health status in the context of general population health surveys. ${ }^{14-17}$

\begin{tabular}{|c|c|c|}
\hline Demographics $(n=6505$ ) & $\begin{array}{l}\text { No recent stressful life events } \\
(26.7 \%)\end{array}$ & $\begin{array}{l}\text { One or more recent stressful } \\
\text { life events }(73.3 \%)\end{array}$ \\
\hline & $\%(95 \% \mathrm{Cl})$ & $\%(95 \% \mathrm{Cl})$ \\
\hline Sex (\% female) & $53.1(51.9,54.3)$ & $55.5(54.3,56.7)$ \\
\hline \multicolumn{3}{|l|}{ Income adequacy } \\
\hline $\begin{array}{l}\text { Lowest } \\
\text { Low-middle }\end{array}$ & $2.0(1.7,2.3)$ & $5.0(4.5,5.5)$ \\
\hline $\begin{array}{l}\text { Low-middle } \\
\text { Middle }\end{array}$ & $6.1(5.5,6.7)$ & $7.9(7.2,8.6)$ \\
\hline Upper-middle & $21.8(20.8,22.8)$ & $21.8(20.8,22.8)$ \\
\hline Highest & $37.8(36.6,39.0)$ & $40.4(39.2,41.6)$ \\
\hline \multicolumn{3}{|l|}{ Education } \\
\hline Less than secondary & $23.4(22.4,24.4)$ & $17.4(16.5,18.3)$ \\
\hline Secondary graduate & $15.1(14.2,16.0)$ & $16.2(15.3,17.1)$ \\
\hline Some post-secondary & $23.5(22.5,24.5)$ & $29.1(28.0,30.2)$ \\
\hline \multirow[t]{2}{*}{ College/university degree } & $37.9(36.7,39.1)$ & $37.3(36.1,38.5)$ \\
\hline & Mean $(95 \% \mathrm{Cl})$ & Mean $(95 \% \mathrm{Cl})$ \\
\hline Age (years) & $48.6(48.2,49.0)$ & $43.1(43.4,44.4)$ \\
\hline Sense of coherence scale (1998) & $64.0(63.7,64.2)$ & $59.4(58.8,60.0)$ \\
\hline Self reported health (1998) & $2.8(2.8,2.8)$ & $2.6(2.6,2.7)$ \\
\hline Self reported health $(2000)$ & $2.7(2.6,2.7)$ & $2.5(2.4,2.5)$ \\
\hline Median number of physician visits (1998) & 2.0 & 3.0 \\
\hline Median number of physician visits (2000) & 2.0 & 3.0 \\
\hline
\end{tabular}


Table 2 Results of the linear regression model predicting self rated health status in 2000

\begin{tabular}{|c|c|c|c|c|c|c|}
\hline \multirow[b]{2}{*}{ Model } & \multirow[b]{2}{*}{$\beta$} & \multirow[b]{2}{*}{ B } & \multicolumn{2}{|c|}{$95 \% \mathrm{Cl}$ for b } & \multirow[b]{2}{*}{ Tolerance } & \multirow[b]{2}{*}{ VIF } \\
\hline & & & Lower & Upper & & \\
\hline (Constant) & & 1.89 & 1.78 & 2.01 & & \\
\hline Age (years) & -0.17 & -0.01 & -0.02 & -0.01 & 0.91 & 1 \\
\hline Sex (referent: male) & -0.03 & -0.06 & -0.10 & -0.02 & 1.00 & 1 \\
\hline SRH in 1998 & 0.49 & 0.52 & 0.49 & 0.54 & 0.87 & 1 \\
\hline RLE in 2000 (referent: none) & -0.05 & -0.12 & -0.17 & -0.08 & 0.92 & 1 \\
\hline SOC in 1998 (centred) & 0.06 & 0.01 & 0.003 & 0.001 & 0.60 & 2 \\
\hline SOC in 1998 (centred) by RLE in 2000 & 0.03 & 0.004 & 0.001 & 0.01 & 0.62 & 2 \\
\hline
\end{tabular}

\section{Analysis strategy}

To accommodate the longitudinal sample weights, the weights were normalised (that is, the weight for each respondent was divided by the average weight) to take into account the probability of selection while controlling for an artificially weight induced increase in sample size.

The distribution of the adjusted recent life events index showed that $60 \%$ of the participants had a score of zero and only $10 \%$ had a score of 2 or more. Because of the extreme skewing of the scores and the interpretability of a dichotomised outcome (that is, did the respondent experience a recent stressful life event?), the scores on the index were dichotomised into zero (no events) or one (one or more life events).

Although some researchers investigating SRH dichotomise the responses (for example, excellent, good and very good compared with fair and poor), there is strong evidence that the responses to global assessments of SRH should be treated as indicators of a continuous construct. ${ }^{18}{ }^{19}$ The hypothesised relation of SOC and the experience of a recent stressful life event on SRH was therefore examined using linear regression analysis (model 1). More specifically, this model regressed SRH, assessed in 2000, onto SOC assessed in 1998, the experience of a recent stressful life event (yes or no) in the 12 months before 2000 (RLE), and a multiplicative interaction term between the two variables. SRH assessed in 1998 was included in the model to control for initial differences in SRH, and age and sex were included as covariates. To address multicollinearity associated with the inclusion of the interaction term, the SOC scores were centred..$^{20}$

Because visits to a medical doctor were recorded as a total count over the previous year, Poisson regression analysis was used to test the hypothesised relation using self reported number of visits to a medical doctor in the previous year as the outcome (model 2). However, in many situations involving individual counts, an unadjusted Poisson model produces misleading estimates of variance terms and misleading inferences about the regression because the

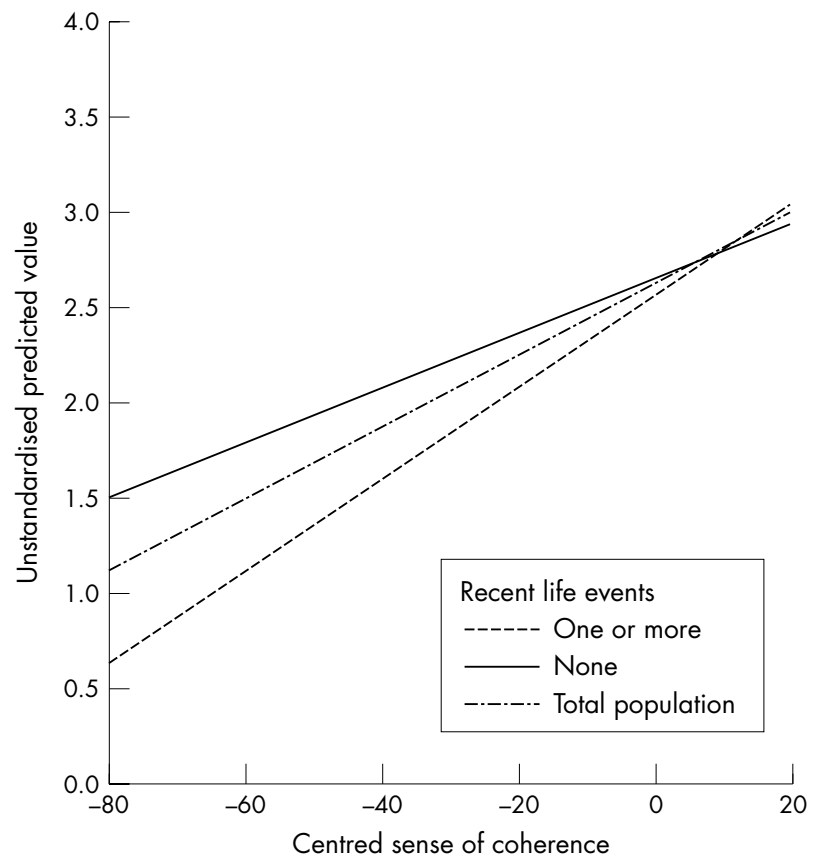

Figure 1 Graph of unstandardised predicted values of self rated health status by centred sense of coherence.

observed counts have larger variances than that implied by the model. This situation, over-dispersion, can be corrected by estimating a dispersion parameter, which is used to rescale the standard errors. ${ }^{21}{ }^{22}$ Specifically, the standard errors of the estimates are multiplied by the square root of the estimated dispersion factor (commonly called the scaling factor). McCullagh and Nelder suggested that it is reasonable to estimate the dispersion parameter using the ratio of the

Table 3 Difference in self reported health status assessed in 2000 across presence or absence of a RLE in the 12 months before 2000 for groups defined as above or below the mean SOC score measured in 1998

\begin{tabular}{|c|c|c|c|c|c|}
\hline \multirow[b]{2}{*}{ SOC $^{*}$ category } & \multirow[b]{2}{*}{ RLE† category } & \multirow{2}{*}{$\frac{\text { SRH } ¥}{\text { Mean }}$} & \multirow[b]{2}{*}{ Mean difference } & \multicolumn{2}{|c|}{$95 \% \mathrm{Cl}$ of the difference } \\
\hline & & & & Lower bound & Upper bound \\
\hline Below mean§ & $\begin{array}{l}\text { None }(n=1772) \\
\text { One or more }(n=948)\end{array}$ & $\begin{array}{l}2.50 \\
2.26\end{array}$ & 0.24 & 0.16 & 0.32 \\
\hline Above mean§ & $\begin{array}{l}\text { None }(n=3003) \\
\text { One or more }(n=793)\end{array}$ & $\begin{array}{l}2.77 \\
2.73\end{array}$ & 0.04 & -0.04 & 0.11 \\
\hline
\end{tabular}

*Sense of coherence assessed in 1998. †Recent stressful life event experienced in the 12 months before 2000. ¥Self rated health status assessed in the 2000. SEqual variances assumed (Levene's test for equality of variances not significant). 
Table 4 Results of the linear regression model predicting self rated health status in 2000 with income adequacy and education level included in the model

\begin{tabular}{|c|c|c|c|c|c|c|}
\hline \multirow[b]{2}{*}{ Model } & \multirow[b]{2}{*}{$\beta$} & \multirow[b]{2}{*}{ b } & \multicolumn{2}{|c|}{$95 \% \mathrm{Cl}$ for $\mathrm{b}$} & \multirow[b]{2}{*}{ Tolerance } & \multirow[b]{2}{*}{ VIF } \\
\hline & & & Lower & Upper & & \\
\hline (Constant) & & 1.89 & 1.78 & 2.01 & & \\
\hline Age (years) & -0.15 & -0.01 & -0.01 & -0.01 & 0.84 & 1 \\
\hline Sex (referent: male) & -0.02 & -0.04 & -0.08 & -0.00 & 0.98 & 1 \\
\hline SRH in 1998 & 0.47 & 0.50 & 0.47 & 0.52 & 0.83 & 1 \\
\hline RLE in $2000^{*}$ (referent: none) & -0.05 & -0.11 & -0.15 & -0.06 & 0.91 & 1 \\
\hline SOC in 1998 (centred) & 0.05 & 0.01 & 0.00 & 0.01 & 0.60 & 2 \\
\hline SOC in 1998 (centred) by RLE in 2000 & 0.02 & 0.00 & 0.00 & 0.01 & 0.62 & 2 \\
\hline Highest level of education & 0.04 & 0.01 & 0.00 & 0.01 & 0.84 & 1 \\
\hline Income adequacy & 0.09 & 0.08 & 0.06 & 0.11 & 0.79 & 1 \\
\hline
\end{tabular}

deviance to its associated degrees of freedom..$^{23}$ Correcting for over-dispersion in this way does not influence the parameter estimates, although it can change the significance levels and accompanying confidence intervals of the model parameters. ${ }^{22} 24$

Measures of socioeconomic status (for example, income and education) were not included in the initial models because they form an integral part of a person's generalised resistance resources and their inclusion in the model would in a sense control for the very effect being investigated. ${ }^{3}$ Although not part of the primary hypotheses, the impact of controlling for education and income, on the moderating effect, was examined after testing the primary hypotheses (that is, model 1 and model 2).

\section{RESULTS}

Table 1 shows specific demographic characteristics of the respondents.

\section{Model 1: self reported health status}

The results of the initial linear regression analysis contained a significant interaction between the experience of a recent stressful life event (RLE) and SOC. However, a review of the tolerance and variance inflation factors (VIF) showed that there was multicollinearity between the main effect of RLE and the SOC-RLE interaction term (the VIF of RLE was 29 and 28 for the SOC-RLE interaction term). ${ }^{25}$ Table 2 shows the results for the regression model using a centred SOC variable (with diagnostics showing that centring SOC had solved the multicollinearity problem).

In addition to reducing multicollinearity, centring can make the interpretation of coefficients more meaningful. As the interaction between recent stressful life events and SOC was significant, the size of the main effect of recent stressful life events depends on the value of SOC. After centring, a value of zero represents the average SOC score and thus the interpretation of the RLE coefficient is conditional on the average SOC score. The RLE coefficient shows the average effect of one or more RLEs on self reported health across all values of SOC. ${ }^{20}$ From the model presented in table 2, the average effect of experiencing one or more RLEs across all values of SOC is a reduction in SRH of 0.12 (on a five point scale ranging from poor to excellent).

To illustrate the interaction, the predicted values for SRH were plotted against SOC separately for both categories of the RLE variable (see fig 1).

Another way of describing the interaction is to compare the mean SRH ratings for groups defined by SOC scores above or below the mean SOC score and the presence or absence of a RLE. From table 3 it can be seen that the mean difference in SRH reported in 2000 between those who did and did not experience a RLE in the 12 months before 2000 was $0.24(t$ $=5.7, \mathrm{p}<0.01$, Cohen's $\mathrm{d}=0.23)$ in people with a below average 1998 SOC and $0.04(t=1.0, \mathrm{p}>0.05)$ in people with a higher than average 1998 SOC score. ${ }^{26}$

Table 4 shows the results after controlling for education and income in the model.

\section{Model 2: visits to a medical doctor}

Table 5 show the results of the Poisson regression analysis. A check of the dispersion factor for the model yielded a value of 6.5 , which showed that the model was over-dispersed (actual dispersion factor $=$ deviance $/ \mathrm{df}=28202.07 / 6498=4.34$; scale parameter equals square root of dispersion factor $=2.08$ ). From table 5 it can be seen that while there were significant main effects for SOC $(t=-6.24, \mathrm{p}<0.01)$ and RLE $(t=21.99, \mathrm{p}<0.01)$, there was no significant moderating effect for SOC (that is, the interaction between SOC and RLE was not significant) $(t=1.1, \mathrm{p}=0.26)$.

\section{DISCUSSION}

A linear regression model was used to test the ability of SOC to moderate (buffer) the impact of experiencing a RLE on future SRH using longitudinal data from the 1998 and 2000

Table 5 Results of Poisson regression analysis predicting number of visits to a medical doctor in 2000

\begin{tabular}{lcrrr}
\hline & Estimate & $\boldsymbol{t}$ Value & Corrected $\boldsymbol{t}$ value & Final $\boldsymbol{p}$ value \\
\hline (Intercept) & 0.43 & 16.98 & 8.16 & $<0.01$ \\
Physician visits in 1998 & 0.05 & 86.85 & 41.75 & $<0.01$ \\
SOC in 1998 (centred) & -0.01 & -12.98 & -6.24 & $<0.01$ \\
Age (years) & 0.01 & 27.84 & 13.39 & $<0.01$ \\
Sex (referent: male) & 0.11 & 8.97 & 4.31 & $<0.01$ \\
RLE* in 2000 (yes/no) & 0.30 & 21.99 & 10.57 & $<0.01$ \\
SOC in 1998 (centred) by RLE in 2000 & -0.002 & -2.34 & 1.13 & 0.26 \\
\hline
\end{tabular}

SOC, sense of coherence. *Recent stressful life event experienced in the 12 months before 2000. Dispersion parameter for Poisson family taken to be 1. Null deviance: 36056.32 with 6504 degrees of freedom. Residual deviance: 28202.07 with 6498 degrees of freedom. 


\section{What this paper adds}

- Recent research in a variety of clinical and work related settings supports Antonovsky's theory that sense of coherence (SOC) moderates (buffers) the health impacts of stressful life events. There is a need for longitudinal, population based studies examining the buffering capacity of sense of coherence.

- By examining longitudinal data from Canada's national population health survey, the results of this study show that SOC buffers the impact of recent stressful life events (for example, family breakdown, financial crisis, physical abuse) on self reported health. Research is needed to explicitly examine the biopsychosocial mechanisms underlying the development and functioning of SOC.

NPHS. The interaction between experiencing a RLE in the 12 months before the 2000 NPHS assessment and SOC, measured two years before (that is, in the 1998 NPHS), was significant. The average effect of experiencing a RLE across all values of SOC was a reduction in SRH of 0.12 . For people with a higher than average SOC, there was no significant impact on SRH associated with the experience of a recent stressful life event in the previous year. However, for people with a below average SOC, the negative impact of experiencing a RLE was an average decline in SRH of 0.24 of a response category (Cohen's $\mathrm{d}=0.23$; a small effect). ${ }^{26}$

Although the limited size of the buffering effect may not be considered important in clinical settings, from a population health perspective the findings represent an additional small but significant piece of the puzzle in the pursuit to understand the determinants of population health. It is worth noting that the effect of experiencing a RLE on SRH seems to be completely mitigated or tempered in people with above average SOC. Additionally, it is plausible that much larger effects could be found if the outcomes examined were more proximal to the experience of stressful life events. For example, SOC might buffer the perceived stress, autonomic nervous system dysregulation, or neuroendocrine response associated with the experience of stressful life events to a much greater extent than was seen with the measure of SRH. Furthermore, the analysis in this study relied on a dichotomised derived index of various RLEs; it is possible that some stressful life events may be buffered to a greater or lesser extent than others in specific populations. A dichotomised variable of occurrence is insensitive to variations in severity. Future research is warranted to evaluate the effects of specific traumatic events, their perceived severity, and the potential for intervention.

Although the primary results of this study provide support for the buffering capacity of SOC, the inclusion of income adequacy and education into the regression model for SRH resulted in a slightly reduced effect of SOC and a nonsignificant interaction effect. This finding supports our initial speculation that income and education contribute to the extent to which one has a pervasive, enduring although dynamic feeling of confidence that (1) the stimuli, deriving from one's internal and external environments in the course of living are structured, predictable, and explicable; (2) the resources are available to one to meet the demands posed by these stimuli; and (3) these demands are challenges, worthy of investment and engagement. Smith et al found that those in semi-skilled and unskilled occupational positions compared with professionals and semi-professionals, those with lower household income, and those with lower job security were significantly more likely to report lower SOC scores. ${ }^{27}$

Although SOC buffered the health impact of RLEs in terms of SRH, it did not buffer the impact on number of medical visits. There are several reasons why the interaction between SOC and recent stressful life events may not have been significant. Although experimentalists frequently find significant interaction effects, non-experimentalists have found the detection of theoretically expected interactions to be difficult. ${ }^{28}$ The difficulty is attributable in large part to the exacerbation of measurement errors and restricted variance (for example, variables with a restricted range) in variables when forming the multiplicative interaction term..$^{28}$

Another reason for the lack of a significant interaction involves the tendency for under-reporting to increase as healthcare utilisation increases. Roberts et al found a pronounced tendency towards greater under-reporting as the total number of visits increases. ${ }^{29}$ Ritter et al also found that people tend to under-report rather than over-report as the use of services increases; no associations were found between demographic or health variables and the size of the reporting error. ${ }^{30}$ However, the model examined in this study controlled for prior utilisation and thus modelled only the factors that influenced any change in utilisation. If the tendency of the participant to under-report did not change substantially from time 1 to time 2 then the under-reporting bias would not be manifest in the analysis. However, if respondents shifted from low to high utilisation rates in response to the experience of a RLE, then the increased tendency to under-report may have resulted in a bias towards the null hypothesis and produced overly conservative estimates of the effect of experiencing a RLE. From this perspective, the impact of the under-reporting bias associated with greater utilisation may have contributed to the lack of a significant interaction in this study.

Although there is some doubt concerning the degree to which the under-reporting bias associated with increased utilisation influenced the results, there is little doubt that the self reports were inaccurate. If the errors in reporting are not associated with specific factors (that is, they can be considered random) then the primary impact of the inaccuracies is an increase in measurement error accompanied by a reduction in power and not the production of biased coefficients. ${ }^{13}$ Although inaccuracies may have contributed to a reduction of power, the large sample size minimised the impact of the random measurement error associated with not finding a significant effect.

In some situations, seeking the services of a physician could be viewed as adaptive coping (for example, seeking help with depression). Given this possibility and the aforementioned methodological issues associated with the interpretation of self reported physician visits, we base our conclusions primarily on the analysis of SRH status. With this in mind, the buffering capacity of SOC seems to be strongly supported. Although studies such as this are limited to observational data lacking random assignment, the addition of prior SRH into the regression analysis should have minimised the impact of such biases (that is, the models can be thought of as explaining a change in health status from 1998 to 2000). The results of studies such as this, combined with growth in knowledge of specific biological mechanisms linking psychosocial characteristics to immune function (for example, Lutgendorf et al and $\mathrm{Coe}^{631}$ ) highlights the need for researchers to take a multidisciplinary perspective in future investigations of Antonovsky's theory of salutogenesis and the broader field of individual differences in resilience to adversity. 


\section{ACKNOWLEDGEMENTS}

Access to the national population health survey data was obtained through the Research Data Centre maintained by Statistics Canada at the University of British Columbia.

\section{Authors' affiliations}

C G Richardson, P A Ratner, School of Nursing, University of British Columbia, Vancouver, Canada

Funding: this research was supported by doctoral fellowships held by $\mathrm{Dr}$ Richardson from the Michael Smith Foundation for Health Research and the Social Sciences and Humanities Research Council of Canada. Dr Ratner is supported by the Canadian Institutes of Health Research.

Conflict of interest: none.

\section{REFERENCES}

1 Kessler RC. A strategy for studying differential vulnerability to the psychological consequences of stress. J Health Soc Behav 1979;20:100-8. 2 Antonovsky A. Health, stress and coping: new perspectives on mental and physical well-being. San Francisco, CA: Jossey-Bass, 1979.

3 Antonovsky A. Unraveling the mystery of health: how people manage stress and stay well. San Francisco, CA: Jossey-Bass, 1987.

4 Antonovsky A. The sense of coherence: an historical and future perspective. Isr J Med Sci 1996;32:170-8.

5 Antonovsky A. The structure and properties of the sense of coherence scale. Soc Sci Med 1993:36:725-33.

6 Lutgendorf SK, Vitaliano PP, Tripp-Reimer T, et al. Sense of coherence moderates the relationship between life stress and natural killer cell activity in healthy older adults. Psychol Aging 1999;14:552-63.

7 Albertsen K, Nielsen ML, Borg V. The Danish psychosocial work environment and symptoms of stress: the main, mediating and moderating role of sense of coherence. Work Stress 2001;15:241-53.

8 Feldt T. The role of sense of coherence in well-being at work: analysis of main and moderator effects. Work Stress 1997;11:134-47.

9 Statistics Canada. National population health survey, 1994-1995 documentation. Ottawa: Statistics Canada, Health Statistics Division, 1995.

10 Statistics Canada. Population health surveys program: national population health survey cycle 4 (2000-2001), household component longitudinal documentation. Ottawa: Statistics Canada, Health Statistics Division, 2002.

11 Statistics Canada. National population health survey, 2000-2001 documentation. Ottawa: Statistics Canada, Health Statistics Division, 2000.

12 Bergner M, Bobbitt RA, Pollard WE, et al. The sickness impact profile: validation of a health status measure. Med Care 1976;14:57-67.
13 Brazier JE, Harper R, Jones NM, et al. Validating the SF-36 health survey questionnaire: new outcome measure for primary care. $B M J$ 1992;305:160-4

14 Benyamini Y, Idler EL. Community studies reporting association between selfrated health and mortality: additional studies, 1995 to 1998. Res Aging 1999;3:392-401.

15 Idler El. Self-assessments of health: the next stage of studies. Res Aging 1999:21:387-91.

16 Idler EL, Benyamini Y. Self-rated health and mortality: a review of twentyseven community studies. J Health Soc Behav 1997;38:21-37.

17 Mossey JM, Shapiro E. Self-rated health: a predictor of mortality among the elderly. Am J Public Health 1982;72:800-8.

18 Manderbacka K, Lahelma E, Martikainen P. Examining the continuity of selfrated health. Int J Epidemiol 1998;27:208-13.

19 Mackenbach JP, van den Bos J, Joung IM, et al. The determinants of excellent health: different from the determinants of ill-health? Int J Epidemiol 1994;23:1273-81.

20 West SG, Aiken LS, Krull JL. Experimental personality designs: analyzing categorical by continuous variable interactions. J Pers 1996;64:1-48

21 Cox DR. Some remarks on overdispersion. Biometrika 1983;70:269-74.

22 Gardner W, Mulvey EP, Shaw EC. Regression analyses of counts and rates: Poisson, overdispersed Poisson, and negative binomial models. Psychol Bull 1995; 118:392-404

23 McCullagh P, Nelder J. Generalized linear models. 2nd ed. London: Chapman and Hall, 1989

24 Pedan A. Analysis of count data using the SAS system. SAS Users Group International (SUGI) Convention, Center Long Beach, CA, 22-25 Apr, 2001. http://www2.sas.com/proceedings/sugi26/p247-26.pdf.

25 Netter J, Wasserman W, Kutner MH. Applied linear statistical models. 3rd ed. Boston, MA: Irwin, 1990.

26 Cohen J. Statistical power analysis for the behavioral sciences. 2nd ed. Hillsdale, NJ: Lawrence Earlbaum, 1988.

27 Smith PM, Breslin FC, Beaton DE. Questioning the stability of sense of coherence: the impact of socio-economic status and working conditions in the Canadian population. Soc Psychiatry Psychiatr Epidemiol 2003:38:475-84.

28 McClelland GH, Judd CM. Statistical difficulties of detecting interactions and moderator effects. Psychol Bull 1993;114:376-90.

29 Roberts RO, Bergstralh EJ, Schmidt L, et al. Comparison of self-reported and medical record health care utilization measures. J Clin Epidemiol 1996:49:989-95

30 Ritter PL, Stewart AL, Kaymaz H, et al. Self-reports of health care utilization compared to provider records. J Clin Epidemiol 2001;54:136-41.

31 Coe CL. Psychosocial factors and psychoneuroimmunology within a lifespan perspective. In: Keating DP, Hertzman C, eds. Developmental health and the wealth of nations: social, biological and educational dynamics. London: Guildford Press, 1999:201-19. 